\title{
Evolution of Multipolar Magnetic Fields in Isolated Neutron Stars and its effect on Pulsar Radio Emission
}

\author{
D. Mitra ${ }^{1}$, S.Konar ${ }^{2}$, D. Bhattacharya ${ }^{1}$, A. V. Hoensbroech ${ }^{3}$, \\ J. H. Seiradakis ${ }^{4}$ and R. Wielebinski ${ }^{3}$ \\ ${ }^{1}$ Raman Research Institute, Bangalore 560080 , India \\ ${ }^{2}$ Inter University Center for Astronomy and Astrophysics, Pune, India \\ ${ }^{3}$ Max-Planck-Institut für Radioastronomie, Auf dem Hügel 69, Germany \\ ${ }^{4}$ University of Thessaloniki, Department of Physics, Laboratory of \\ Astronomy, GR-54006 Thessaloniki, Greece
}

\begin{abstract}
The evolution of the multipolar structure of the magnetic field of isolated neutron stars is studied assuming the currents to be confined to the crust. Lower orders $(\leq 25)$ of multipole are seen to evolve in a manner similar to the dipole suggesting little or no evolution of the expected pulse shape. We also study the multifrequency polarization position angle traverse of PSR B0329+54 and find a significant frequency dependence above $2.7 \mathrm{GHz}$. We interpret this as an evidence of strong multipolar magnetic field present in the radio emission region.
\end{abstract}

\section{Introduction}

Strong multipolar components of the magnetic field have long been thought to play an important role in the radio emission from pulsars. Multipolar components present even in a small degree can significantly change the radius of curvature of the field lines near the polar cap and determine the illumination of the pulsar beam. This in turn might be responsible for the observed complexity in pulse profiles. Significant evolution in the structure of the magnetic field may therefore lead to simplification of pulse profile with age. We explore the ohmic evolution of multipolar magnetic fields in isolated neutron stars assuming the flux to be confined within the crust.

Multipolar magnetic fields would also cause the polarisation position angle (PPA) traverse to depart from the well known rotating vector model of Radhakrishnan and Cooke (1969) (RC). As a result of radius-to-frequency mapping (RFM) radio emission at higher frequencies originate closer to the stellar surface where multipole fields are stronger. This would lead to a frequency dependence of the PPA traverse, departures from the RC model being stronger at higher frequencies. We monitor the PPA of PSR B0329+54 at several frequencies to investigate this effect.

\section{Evolution of Multipolar Magnetic fields}

In order to calculate the evolution of the multipolar magnetic field we solve the ohmic diffusion equation numerically. We assume the currents responsible for the entire field structure to be confined to the crust of the neutron star. We 

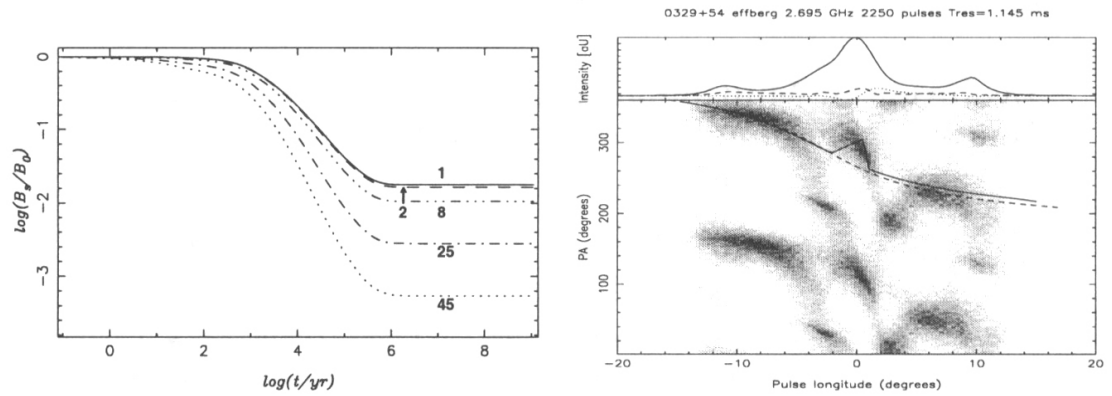

Figure 1. The figure on the left shows the evolution of various multipole orders due to ohmic diffusion. The curves are labeled with multipole orders. The figure on the right shows a grey scale representation of the PPA of PSR B0329+54 at $2.7 \mathrm{GHz}$ (we have used archival data obtained using the Efflesberg Radio Telescope). The dotted line denotes the dipole $\mathrm{RC}$ curve as obtained at lower frequencies. The continuous line models the PPA traverse including a quadrupole component.

adopt a well motivated choice for the electrical conductivity as a function of depth in the neutron star interior and use a standard cooling curve to represent the evolution of temperature (for details see Mitra et al. 1999).

Fig. [1] (left panel) shows the evolution of different multipole orders assuming the same initial strength for all orders. It is evident from the figure that except for very high orders the reduction in the field strength is similar to that of a dipole. Hence no significant evolution of the pulse shape is expected due to the evolution of the multipolar structure of the field.

\section{Polarization position angle of PSR B0329+54}

We investigate the multifrequency behaviour of the polarization position angle of PSR B0329+54 at a number of frequencies over a wide range (from $408 \mathrm{MHz}$ to $4.8 \mathrm{GHz}$ ). The PPA curves at $408 \mathrm{MHz}$ and $1.4 \mathrm{GHz}$ are in agreement with the dipolar RC model but at $2.7 \mathrm{GHz}$ and $4.8 \mathrm{GHz}$ the PPA appears to have developed a kinky feature (Fig. [1] right panel) which is absent at lower frequencies. Assuming RFM, the observed kinkiness might be due to the presence of nondipolar magnetic fields in the emission region. The continuous line as shown in Fig. [1] is a model for the nondipolar magnetic field structure (a combination of a dipole and a quadrupole located at the center of the star) which provides a good fit to the observed PPA (Mitra 1999).

\section{References}

Mitra, D., 1999, PhD Thesis submitted to Jawaharlal Nehru University, New Delhi

Mitra, D., Konar, S. \& Bhattacharya, D., 1999, MNRAS, 307, 459

Radhakrishnan, V. \& Cooke D. J., 1969, Astrophysics Letters, 3, 225 